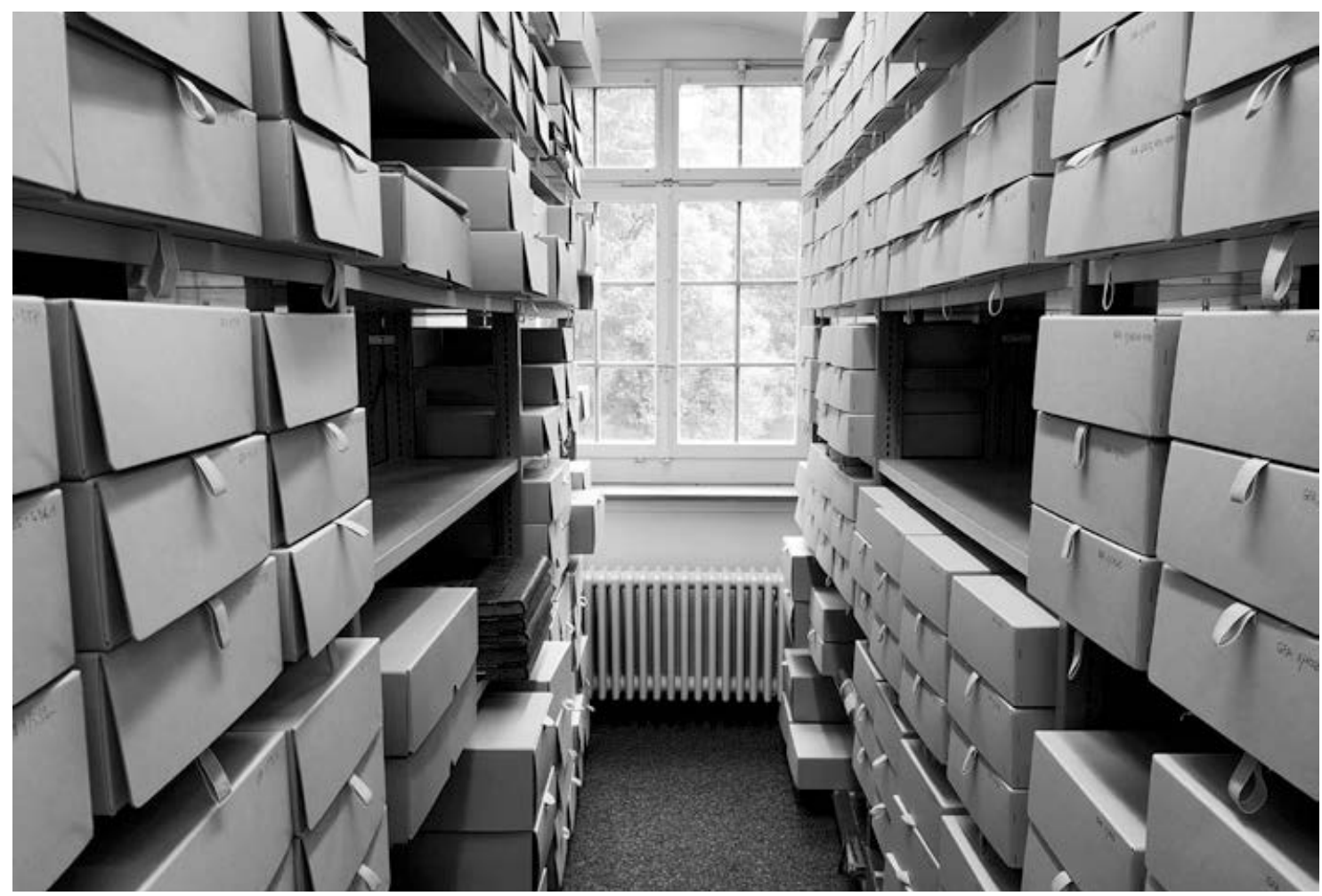

Abb. 1 Blick in Regale des Konzernarchivs der Georg Fischer AG Schaffhausen, Juni 2019, Arbeitsplatz einer unserer Gesprächspartnerinnen

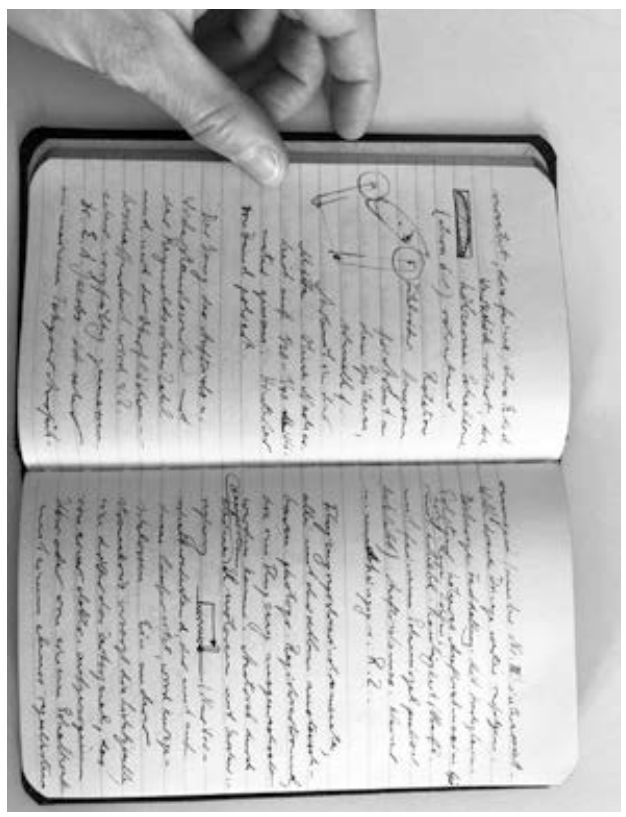

Abb. 2 Archivfund Reisetagebuch 


\section{ARCHIVREISEN \\ Teil I: Zuhause im Archiv}

von DANIELA ZETTI / HANNAH ZINDEL

1 Vgl. Hansjörg Bay, Wolfgang Struck: Vorwort, in: dies. (Hg.): Literarische Entdeckungsreisen. Vorfahren - Nachfahrten - Revisionen, Köln 2012, 9-14, hier 10.

2 Wir haben bislang drei Gespräche geführt, die wir für diesen Text erstmals auswerten. Weitere Interviews mit Archivar_innen und mit Forschenden verschiedener Disziplinen sind in Planung.
Reiseberichte sind unter Archivforscher_innen beliebt. Sie sind meist persönlich und stammen aus erster Hand. Publiziert werden sie nur selten. Vielmehr sind sie Teil eines vielgestaltigen Produzierens von Wissen. Zu diesem gehören das Notieren und Aufzeichnen, das Aufbauen von Ablagesystemen oder das publizistische Aufbereiten von Forschungsergebnissen. Erzählungen über Archivrecherchen folgen narrativen Mustern, die aus historischen Reiseberichten bekannt sind. In ihnen verknüpfen Reisende Entdeckungen und Begegnungen, Eroberungswillen und Lust am Sich-Verlieren, Rationalität und Ekstase. ${ }^{1}$ Sie betonen zudem das Regelhafte des Forschens, reflektieren Wissensordnungen und geben methodische Herangehensweisen weiter. In Archivreiseberichten benennen Forscher_innen Routinen wissenschaftlichen Arbeitens.

Der vorliegende Essay greift Aussagen von Forscher_innen und Archivar_innen auf, die wir in den letzten Wochen bei Online-Interviews gesammelt haben. ${ }^{2}$ In den Gesprächen kamen drei unterschiedliche Perspektiven auf Archivforschung zum Ausdruck: die einer Archivnutzerin, die einer Archivarin und die eines Historikers, der ein Archiv mit aufbaut. Wie prägen digitale Werkzeuge Archive? Wie verändern sich die Reise ins Archiv und die Produktion von Wissen im Archiv, wenn Infrastrukturen, Kommunikation und Dokumente im und um das Archiv herum digital werden? Braucht man noch Archivreisen? Dieser Text ist der erste von zwei Essays zur Archivreise. In ihm verknüpfen wir einleitend Forschungsliteratur zu Entdeckung und Zufall, zur Reise und zum Archiv, um anschließend Formen des eigenen Archivs, des bome 
archive und des Privatarchivs zu beleuchten. Im nächsten Essay widmen wir uns Figurationen von Schutz und Gastlichkeit im Archiv.

\section{Vom Suchen und Finden}

Das Reflektieren über wissenschaftliche Arbeit folgt bisweilen einem eher unterhaltsamen Stil. Sprecher_innen setzen sich bewusst über bekannte akademische Formalismen hinweg, wenn sie sich über eine Situation in der Lehre oder eine Konferenz austauschen. Archivreiseberichte zeichnen sich gegenüber solchen Rede- und Reflexionsweisen allerdings durch die Beschreibung einer doppelten Bewegung aus: Sie handeln von der Reise zum Archiv und von jener durch das Archiv. ${ }^{3}$

In Stil und Funktion sind Archivreiseberichte mündliche Verwandte jener Reiseberichte, die im I 8. und I9. Jahrhundert Narration und Systematik, Apodemik und Forschung nebeneinanderstellten. ${ }^{4}$ Expeditionen, die bekanntesten raumgreifenden Suchbewegungen der Wissenschaft, haben wissenschaftliche und literarische Textproduktion gleichermaßen angeregt. ${ }^{5}$ Archivreisen werden zwar in der (Forschungs-)Literatur selten als Erkundungsreisen beschrieben. ${ }^{6}$ Doch auch Archivreisen aktivieren Weltwissen, warten mit Abenteuern auf und stellen Reisende vor Aufgaben, die sie in ihrem Organisationsgeschick herausfordern. ${ }^{7}$

Archivreisen lassen sich zudem im Anschluss an Forschungsliteratur aus der Wissensgeschichte betrachten. So handeln sie gern von der Trouvaille, von dem einen krönenden Zufallsfund, der alle Erwartungen gesprengt und einstige Tatsachen umgestoßen hat. ${ }^{8}$ Der Zufall, der entscheidende Entdeckungen vermeintlich erst möglich macht, ist auch ein stabiler Gemeinplatz der Geschichte naturwissenschaftlicher Entdeckungen und technischer Erfindungen. Wer von seiner Macht berichtet, kann komplexe technische, bauliche und soziale Arrangements des Forschens und Erkennens ausblenden. Er_Sie kann die sonst so präsente Bedeutung von Methode und «Denkstil〉 für die Entstehung wissenschaftlicher Tatsachen ausnahmsweise unterschlagen. ${ }^{9}$ Eine wichtige Funktion der Rede vom Zufall liegt für die Wissenschaft darin, das schwer benennbare Ziel des Forschens zu überliefern: die Suche nach dem genuin Unbekannten. In den Worten Hans-Jörg Rheinbergers: «Was wirklich neu ist, ist definitionsgemäß nicht vorhersehbar, es kann also auch nicht gezielt herbeigeführt werden. Was wirklich neu ist, muss sich einstellen, es können nur Rahmenbedingungen dafür geschaffen werden, dass es sich ereignen kann.» ${ }^{10}$

Der Ort des Archivs schließlich ist prekär: In den Kulturwissenschaften ist das Archiv als ein Erinnerungsort zur Aufbewahrung und Produktion von Vergangenheit beschrieben worden. ${ }^{11}$ In der Wissenschaftsgeschichte war vom archival turn die Rede. ${ }^{12}$ Gefragt wurde nach Formen des Archivierens in den Geistes- und Naturwissenschaften sowie nach den Grenzen des
3 Zum Verhältnis von Reisen und Forschen vgl. Philippe Despoix: Die Welt vermessen. Dispositive der Entdeckungsreise im Zeitalter der Aufklärung, Göttingen 2009.

4 Vgl. Christian von Zimmermann: Vorwort, in: ders.: Wissenschaftliches Reisen - reisende Wissenschaftler. Studien zur Professionalisierung der Reiseformen zwischen 1650 und 1800 , Heidelberg 2002, 7-18, hier 14. Die Ausdifferenzierung der Wissensbereiche im 18. Jahrhundert führte zu einer Spezialisierung, Professionalisierung und Institutionalisierung des Reisens und des Reiseberichts.

5 Vgl. Hansjörg Bay, Wolfgang Struck: Forschungsreise. Exploration of a Genre, in: dies. ( $\mathrm{Hg})$ : Literarische Entdeckungsreisen, 15-26.

6 Ausnahmen bestätigen die Regel, z. B. Kate Grenville: Searching for the Secret River, Edinburgh u. a. 2007.

7 Zum Weltwissen vgl. Iris Schröder: Die Erde im Archiv. Das Projekt einer Humangeographie in Bildern, 1911-1931, in: dies., Sabine Höhler (Hg.): Welt-Räume. Geschichte, Geographie und Globalisierung seit 1900, Frankfurt / M., New York 2005, 100-116.

8 Zum sogenannten Vetorecht der Quellen vgl. Reinhart Koselleck: Standortbindung und Zeitlichkeit. Ein Beitrag zur historiographischen Erschließung der geschichtlichen Welt, in: ders., Wolfgang J. Mommsen, Jörn Rüsen (Hg.): Objektivität und Parteilichkeit in der Geschichtswissenschaft, München 1977, 17-46.

9 Vgl. Ludwik Fleck: Entstehung und Entwicklung einer wissenschaftlichen Tatsache. Einführung in die Lehre vom Denkstil und Denkkollektiv, Frankfurt/M. 1980 [1935].

10 Hans-Jörg Rheinberger: Über Serendipität - Über Forschen und Finden, in: Orlando Budelacci: Imagination. Suchen und Finden, Paderborn 2014, 233-243.

$11 \mathrm{Vgl}$. Aleida Assmann: Archive im Wandel der Mediengeschichte, in: Knut Ebeling, Stephan Günzel (Hg.): Archivologie. Theorien des Archivs in Philosophie, Medien und Künsten, Berlin 2009, 165-175.

12 Vgl. Kasper Risbjerg Eskildsen: Leopold Ranke's Archival Turn. Location and Evidence in Modern Historiography, in: Modern Intellectual History, Bd. 5, Nr. 3, 2008, 425-453. 

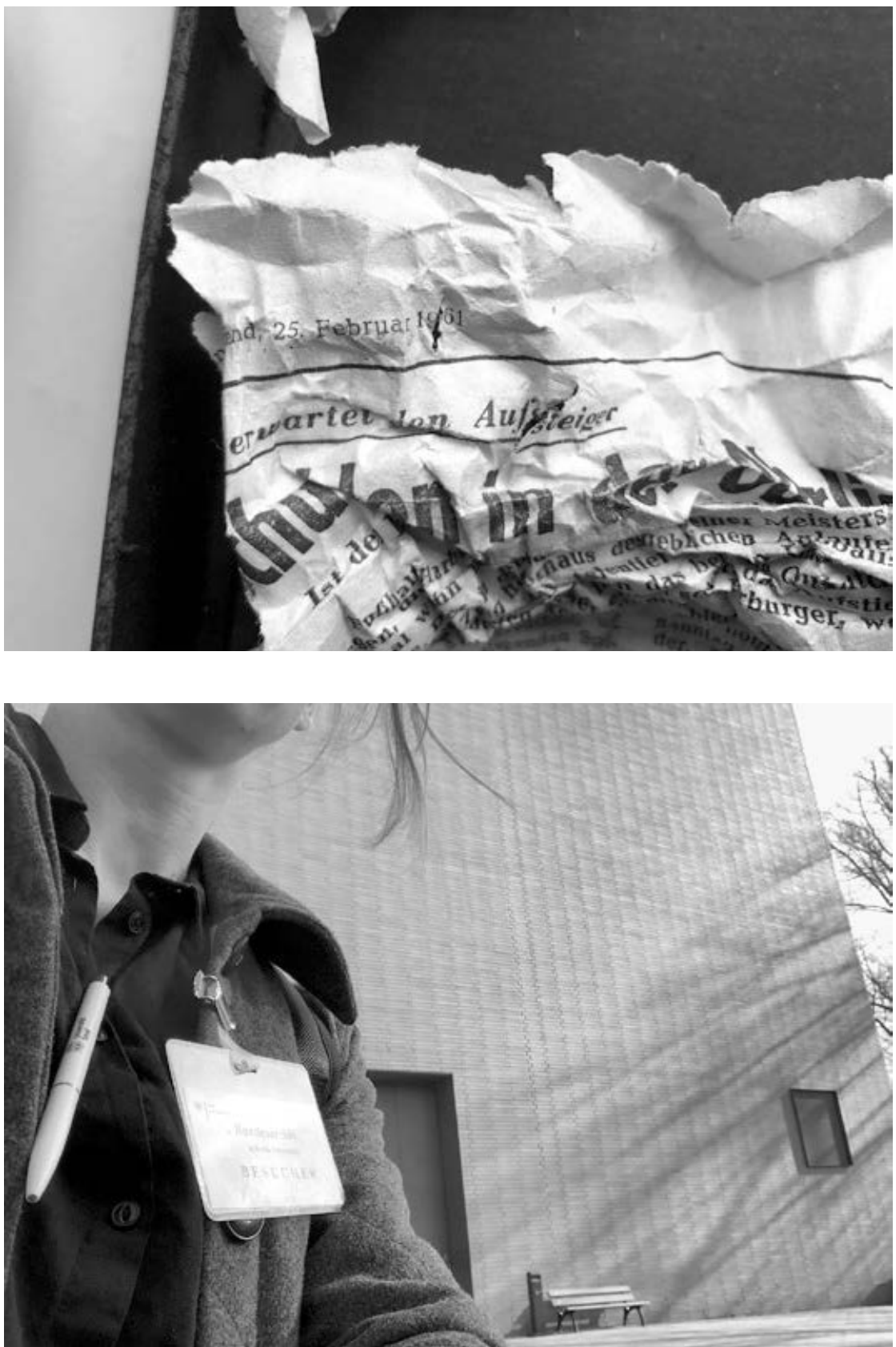

Abb. 3 Die Zeitungsverpackung hilft beim Datieren einer Filmrolle.

Abb. 4 Ausweis für Besucher_innen des Bundesarchivs 
Archivs. ${ }^{13}$ An der Schnittstelle von Geschichte und Medienwissenschaft rückten derweil sensorische, materielle und institutionelle Aspekte des Archivierens in den Blick. Diese erfahren auch in mündlichen Archivreiseberichten stärkere Geltung als in den nüchternen Bestandsbeschreibungen, mit denen geschichtswissenschaftliche Monografien eingeleitet werden. ${ }^{14}$ Archive wurden als Schatzkammern betrachtet, gefüllt mit rostigen Klammern und rauen Kordeln, ${ }^{15}$ und auf Zugänglichkeit geprüft. ${ }^{16}$ Mit dem Fokus auf Technologien und Praktiken des Speicherns und Übertragens ${ }^{17}$ wurde in der historischen Medienwissenschaft die Forderung laut, das Internet selbst müsse stärker als Archiv genutzt werden. ${ }^{18}$ Doch wie ist die Lage des Archivs, wenn es als Reiseziel betrachtet wird?

\section{Die Regel vom <feuchten Daumen>}

Die Regel vom <feuchten Daumen> ist metaphorisch und meint das Durchblättern und Überfliegen von Informationen, das beiläufige und doch hochkonzentrierte Identifizieren des Bemerkenswerten. Sie ist die erste und vornehmste aller Archivregeln. Ein Besuch ist ein zeitlich begrenzter, kein Daueraufenthalt. Es soll schnell gehen. Informationen müssen von den Nutzer_innen ausgewählt und nach Hause geschafft werden. Archivalien sind nicht ausleihbar wie die Bücher einer Bibliothek. Im Archiv werden auch keine Bücher geschrieben. Es gilt, Informationen mobil zu machen, die an einen festen Ort gebunden sind. Dazu braucht es Transkripte, Notizen, Fotografien, Kopien und Scans. Technisch und physisch kann es herausfordernd sein, Archivmaterial in Bewegung zu setzen. ${ }^{19}$

«Wie krieg ich jetzt die Information von dort nach Hause?» ${ }^{20}$ ist entsprechend eine Frage, die sich im Archiv wohl jede_r schon einmal gestellt hat. Die Medienhistorikerin Sarine Waltenspül empfiehlt Archivbesucher_innen, zu einem frühen Zeitpunkt im Forschungsprozess «lieber alles zu fotografieren, auch das scheinbar Falsche». Dazu nimmt sie manchmal einen magic arm mit ins Archiv, also eine Kamerahalterung mit Schiene. Später lasse sich in den Fotografien dann noch viel mehr entdecken, nachträgliche Aufbereitung lohne sich. Sie bezeichnet das als «immer wieder ins eigene Archiv gehen». ${ }^{21}$ Besitzen Forschende oft Ordner voller Digitalfotos und hochaufgelöste Scans oder mehrere Kilogramm schwere Pakete mit Kopien, so finden bei Archivar_innen bisweilen Dubletten den Weg in Privatwohnungen. Die Archivarin Franziska Eggimann mag deshalb den von uns eingebrachten Begriff des home archive. «Gerade Sachen, die ich für Anfragen sehr oft verwende, um eben auch selber zu recherchieren», so Eggimann, hätten lange Zeit als Dubletten bei ihr zu Hause im Regal gestanden. ${ }^{22}$ Als mit den ersten Corona-Maßnahmen aus ihrem einen regulären Tag Home-Office pro Woche mehr wurden, bewährte sich allerdings die seit acht Jahren vorangetriebene Digitalisierungsstrategie ihres Archivs. Diese erlaubte ihr auch von
13 Vgl. Lorraine Daston: Science in the Archives. Past, Presents, Futures, Chicago, London 2017.

$14 \mathrm{Vgl}$. Mario Wimmer: Archivkörper. Eine Geschichte historischer Einbildungskraft, Konstanz 2012.

$15 \mathrm{Vgl}$. Arlette Farge: Der Geschmack des Archius, Göttingen 2018 [1989].

16 Vgl. Cornelia Vissmann: Das Recht und seine Mittel. Ausgewählte Schriften, Frankfurt/M. 2012, 181-188.

17 Vgl. Jörg Paulus: Archiv. Zur Einführung, in: Andreas Ziemann ( $\mathrm{Hg}$.): Grundlagentexte der Medienkultur. Ein Reader, Wiesbaden 2019, 79-82.

18 Vgl. Niels Brügger: The Archived Web. Doing History in the Digital Age, Cambridge 2018.

19 Die Regel vom feuchten Daumen wird in geschichtswissenschaftlichen Proseminaren vermittelt. Archivar_innen warnen hingegen bisweilen davor, den Daumen tatsächlich mit Speichel zu befeuchten, um leichter umblättern zu können.

20 Interview mit Christian

Bunnenberg am 30.4.2021, Min oo:18:20.

21 Interview mit Sarine Waltenspül am 28.4.2021, Min 00:17:40.

22 Interview mit Franziska Eggimann am 21.4.2021, Min o0:19:00. 
23 Ebd., o0:16:30.

24 Ebd., o0:38:00.

25 Ebd., oo:26:15.

26 Ebd., o0:49:38.

27 Interview mit Christian

Bunnenberg, 00:30:30 und 00:31:00.

28 Siehe coronarchiv.blogs.

uni-hamburg.de.

29 Interview Bunnenberg, oo: $46: 48$.

30 Philipp Felsch: 14.777 Dinge. Verkehr mit der Sammlung Schlagintweit, in: Friedrich Balke u. a. (Hg.): Die Wiederkehr der Dinge, Berlin 2011, 193-207, hier 195.

31 Hannah Zindel: Ballons. Medien und Techniken früher Luftfahrten, Paderborn 2020, 45.

32 Bernd Stiegler: Reisender Stillstand. Eine kleine Geschichte der Reisen im und um das Zimmer herum, Frankfurt/M. 2010, 3. Gemeint ist hier die Zimmerreise sowohl im Sinne einer "Erkundung der Nähe» oder einer Erfahrung der «Fremdheit des Vertrauten" (ebd., 52) als auch im Sinne von Spaziergängen durch "eine eigens konstruierte virtuelle Welt» (ebd., 263). daheim, «mit den Materialien [zu] arbeiten». ${ }^{23}$ Für Eggimann ist allerdings klar, dass das Forschen in digitalen Beständen Vorkenntnisse über das Archiv, seine Bestände und Dokumente verlangt. Man sollte diese im Idealfall schon erkundet haben - also Korpus, Formate und Materialitäten kennen -, bevor man digital recherchiert. «Digital ist sehr hilfreich, wenn du die Thematik oder das Archiv oder die Unterlagen schon recht gut kennst. [...] Aber sonst digital zu recherchieren, find ich sehr, sehr schwierig», erklärt sie. ${ }^{24}$ Digitale Nutzung äußert sich in ihrem Archiv «durch mehr Kontakt, mehr Anfragen, ja überhaupt Sichtbarkeit». ${ }^{25}$ Besucher_innen können ihrer Meinung nach Archive sehr gut kennenlernen, sie können ihnen «zweite Heimat» werden. ${ }^{26}$

Der Historiker Christian Bunnenberg schildert weitere Varianten des Archivierens zuhause. In seiner Studienzeit hat er das «Privatarchiv» eines bekannten Stern-Journalisten als «völliges Faszinosum» und «Wundertüte» wahrgenommen: ${ }^{27}$ eine Wohnung in Hamburg-Altona, in der ausnahmslos alle Wände bis unter die Decke mit Regalen voller schwarzer Aktenordner zugestellt waren, darin Dinge in einzelnen Klarsichthüllen. Die Lagerung gesammelter Dinge beschäftigt den Juniorprofessor zudem, seit er im März 2020 Mitbegründer des sogenannten coronarchivs wurde, das Fotos von Objekten und Situationen sowie Texte mit Pandemiebezug sammelt. ${ }^{28}$ Auf den Servern der Uni Hamburg gehostet, soll es mithilfe eines Eingabeformulars und einer Upload-Funktion «jeder und jedem die Möglichkeit geben, Alltagsbeobachtung als Spur in digitaler oder digitalisierter Form zu hinterlassen». ${ }^{29}$ Digital, denn: Er habe keine Halle, in die er Sachen einlagern könne.

Das eigene Archiv, das home archive und das Privatarchiv unserer Interviewpartner_innen sind Hybride zwischen Wohn-, Arbeits- und Archivräumen. Betrachtet man sie genauer, rücken Techniken und Praktiken der Nachbereitung intensiver Sammlungsaktivität in den Fokus. Philipp Felsch hat am Beispiel der Asienreise der Brüder Schlagintweit gezeigt, wie zuhause aus den «sprechenden Objekten» einer Reise «Requisiten, Zeug und Gerümpel» werden und aus Reisenden «traurige Humboldtianer«, die «unter der Last der Dinge», unter Aufschub, Verzettelung und Verzögerung nach der Reise leiden. ${ }^{\mathbf{3 0}}$ Digitale Sammlungen, wie die unserer Gesprächspartner_innen, bieten hingegen die Möglichkeit, immer wieder zu reisen und zu entdecken und auch mal mit trockenen Fingern zu scrollen. Sowohl das eigene digitale Arbeitsarchiv als auch das coronarchiv lassen sich zwar nicht betreten, sind aber dank einer in steten Bearbeitungsschritten erfolgten Annäherung und Aneignung bereisbar. Es gibt wie bei einer «Kartenreise» ${ }^{31}$ oder einer «Zimmerreise» ${ }^{32}$ immer wieder Neues im Bekannten zu entdecken. Verloren gehen kann man natürlich weiterhin. Auch wenn die Digitalisierung von Dokumenten, Software sowie Online-Suchhilfen den Nutzenden eine Brücke bauen für diese Reisen ins Archiv - das bome archive verdankt sich nicht der Digitalisierung, und das coronarchiv verdankt sich nicht Corona. Sie entstehen aus der Erwartung heraus, 
dass sich das, was im Sinne Hans-Jörg Rheinbergers <wirklich neu ist> für die Forschung, vielleicht erst nach der Pandemie, auf dem Weg ins nächste Archiv, zuhause oder auf einem Spaziergang einstellt.

\section{Zwischenstation}

Der Blick auf die Archivreise verschiebt den Fokus von der festen Adresse zur Zwischenstation. In den Vordergrund treten so Praktiken und Techniken des Arbeitens im Archiv, wie der magic arm, der feuchte Daumen und das Paket mit Kopien. Archivreiseberichte sind Momentaufnahmen des Über-Setzens: von der Quelle zum Buch, aus der Vergangenheit in die Wissenschaft und gegenwärtig immer auch aus Institutionen in die Ordnung des Digitalen. 